\title{
Build The Future - Gravity Dams: A Primeira Versão de um Aplicativo Mobile para Ensino de Estabilidade de Barragens à Gravidade
}

\author{
Bruno T. L. Colodel ${ }^{1}$, Arthur M. de Oliveira ${ }^{2}$, Andreia de Jesus ${ }^{2}$ \\ ${ }^{1}$ Departamento de Hidráulica e Saneamento - Universidade Federal do Paraná (UFPR) \\ Centro Politécnico, Bloco V, $1^{\circ}$ andar - Jardim das Américas - Curitiba, PR - Brasil \\ ${ }^{2}$ Setor de Educação Profissional e Tecnológica - Universidade Federal do Paraná \\ (UFPR) - Curitiba, PR - Brasil \\ bruno.colodel@ufpr.br, arthurmattoso@gmail.com, aj ufpr@yahoo.com
}

\begin{abstract}
The unsatisfactory performance of Civil Engineering courses in national assessment exams opens up space for discussion of ways to facilitate the assimilation of the content by the students. The use of new technologies in teaching helps to meet this demand. In this sense, this work presents an educational software for mobile devices to be used by teachers in teaching content about gravity dams. A structure of learning modules was implemented, containing conceptual and simulation chapters. The evaluation of this software obtained good results both in the view of a specialist professor in the area and in the view of an information technology analyst.
\end{abstract}

Resumo. O desempenho pouco satisfatório dos cursos de Engenharia Civil nos exames de avaliação nacionais abre espaço para discussão sobre formas de facilitar a assimilação do conteúdo por parte dos alunos e alunas. $O$ uso de novas tecnologias no ensino auxilia no atendimento a esta demanda. Neste sentido, o presente trabalho apresenta um software educativo para dispositivos móveis a ser utilizado pelos docentes no ensino do conteúdo sobre barragens à gravidade. Foi implementada uma estrutura de módulos para aprendizado, contendo capítulos conceituais e de simulação. A avaliação deste software obteve bons resultados tanto na visão de um professor especialista na área quanto na visão de um analista de tecnologia da informação.

\section{Introdução}

O rápido avanço das tecnologias digitais demanda profissionais qualificados não apenas em sua área específica, mas também que saiba aliar tecnologias de modo a atender as necessidades do mercado de trabalho. Devido a isto, hoje, as instituições de ensino têm cada vez mais se modernizado para atender esta tendência. No caso da engenharia civil, um dos cursos mais antigos das universidades brasileiras, este processo é moroso, mas necessário.

E esta necessidade se reforça com base nos resultados alcançados no ENADE. Apesar do número de formados em engenharia disparar nos últimos anos, a qualificação destes profissionais têm sido um entrave. Segundo o último ENADE realizado na área 
das engenharias, em 2019, e considerando uma escala de desempenho de 0 a 100 , os cursos de engenharia não obtiveram médias satisfatórias. A engenharia civil obteve 39,9; a engenharia mecânica ficou com 36,4; e a engenharia elétrica obteve o pior resultado entre as engenharias avaliadas com 33,4 [Ministério da Educação 2019].

Mas, já se encontram na literatura, antes mesmo dos resultados do ENADE de 2019, pesquisas que se preocupam em aprimorar o processo de ensino-aprendizagem em cursos de engenharia. Um exemplo é o estudo realizado por Pereira (2005), na disciplina de Engenharia de Transportes da Universidade de São Carlos, que coloca que o maior desafio em termos de qualidade de ensino em engenharia é a incorporação das mudanças tecnológicas e sociais. Utilizando-se do construtivismo de Jean Piaget, Pereira (2005) chegou à conclusão de que a introdução dos conteúdos da disciplina num site da Internet aumentou significativamente o interesse dos alunos e alunas e notas $11 \%$ maiores. Outro exemplo é o trabalho de Trevisan e Silva (2019), que implementaram uma metodologia ativa de aprendizagem chamada de Peer Instruction, proposta pelo professor de física da Universidade de Harvard Eric Mazur e, também, inspirada no construtivismo. Os alunos que obtiveram resultados acima da média para aprovação representaram $95 \%, 100 \%$ e $91 \%$ do total de alunos em cada turma analisada com esta técnica, contra $45 \%, 64 \%$ e $33 \%$ nas turmas em que se utilizou o método tradicional de ensino. Segundo os autores, o sucesso se deu pela troca de informações entre os discentes o que possibilitou o desenvolvimento da análise crítica e da capacidade de ensinar e aprender.

É importante colocar que a teoria de aprendizagem de Piaget, o construtivismo, conforme Abreu et. al (2010) reforçam em seu trabalho, a ênfase está na reconstrução dos caminhos pelos quais o indivíduo evoluiu de modo que o conhecimento é um processo se estruturando e não um estado já cristalizado. E essa reconstrução e reanálise de conteúdos é essencial para o sucesso na aprendizagem de muitos conteúdos das áreas das engenharias, como por exemplo, o conteúdo sobre barragem à gravidade.

E para o desenvolvimento de um projeto de uma barragem à gravidade é necessário que o engenheiro civil realize uma série de cálculos de estabilidade, a fim de garantir que esteja nos padrões de segurança estabelecidos pelos Critérios de Projeto Civil da Eletrobrás [Eletrobrás 2003]. Estes cálculos são de alta complexidade, exigindo muito aprendizado para que se domine o conteúdo. Portanto, uma ferramenta que disponibilize tutoriais, simulações e desafios para auxiliar os alunos e alunas (e engenheiros(as) formados) é de grande valia para atingir os objetivos de ensinoaprendizado deste tema tão relevante na formação de acadêmicos de engenharia civil.

Portanto, o objetivo deste artigo é apresentar a primeira versão de um aplicativo mobile para ensino de estabilidade de barragens à gravidade. Desta forma, disponibilizar um software educativo para auxiliar no processo de ensino-aprendizagem de conceitos acerca de estabilidade de seções com largura unitária e comuns de barragens à gravidade, demonstrando o passo a passo das análises e simulações decorrentes de cada resultado encontrado. E, com isso, disponibilizar mais uma metodologia de ensino para esse conteúdo, que seja menos complicada, mais ágil e até divertida. 


\section{Base Teórica e Trabalhos Correlatos}

As barragens são estruturas que aprisionam substâncias líquidas ou misturas de líquidos e sólidos em curso permanente ou temporário de água [Brasil 2010]. Podem variar de pequenos maciços a enormes estruturas de concreto que são geralmente utilizadas para irrigação, fornecimento de água e geração de energia elétrica [Coelho 2016].

As barragens à gravidade são aquelas formadas por grandes maciços de concreto. Apesar de possuírem limitação de altura com relação à base e à fundação, requerem pouca manutenção e se adaptam em qualquer localidade, dependendo, porém, da fundação do local. Elas são dependentes de sua massa para estabilidade e o perfil de gravidade é geralmente trapezoidal para evitar sobrecarga (Figura 1).
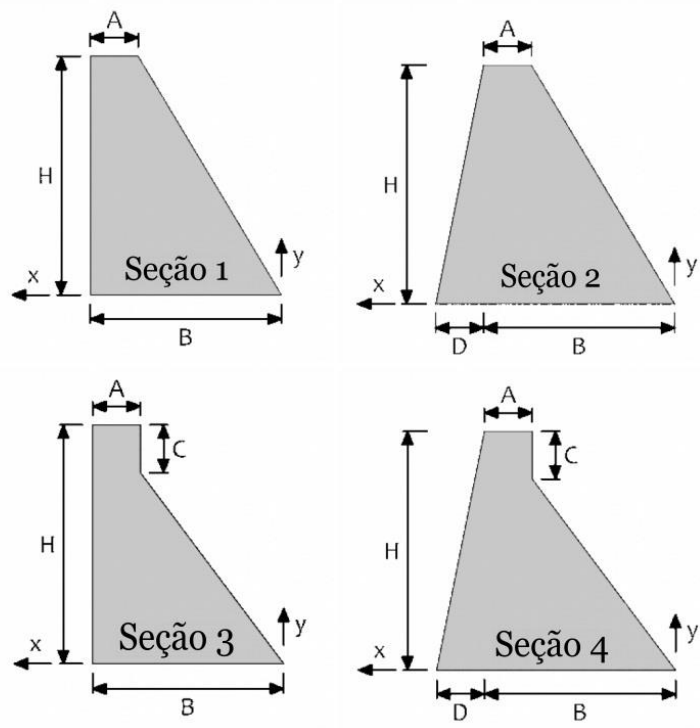

Figura 1. Seções comuns de barragens à gravidade [Coelho 2016]

Elas devem ser dimensionadas considerando combinações de cargas que incidem permanentemente ou ocasionalmente na estrutura, considerando um fator de segurança adequado a cada uma. Para cada combinação são calculados coeficientes de segurança quanto à flutuação, tombamento, deslizamento e tensões na base [Coelho 2016].

O cálculo desses coeficientes é bastante complexo. Portanto, é necessária uma metodologia de ensino que permita ao aluno desenvolver este conhecimento durante o processo de ensino-aprendizagem, por meio de testes e análises dos resultados. Logo, considerando os estudos de Pereira (2005) e Trevisan e Silva (2019), citados na Seção 1, o aplicativo mobile Gravity Dams também se consolidou nas bases teóricas do construtivismo de Piaget. Além disso, considerando a natureza do conteúdo sobre barragens à gravidade, é necessário que o(a) aluno(a) se envolva com esse conteúdo por meio da elaboração de hipóteses, leituras e discussões para validar sua compreensão. E, conforme classificação de Valente (1999), o tipo de software simulação permite ao aluno(a) alterar certos parâmetros e observar o comportamento de um fenômeno/conteúdo. Isto é, o aprendiz não escolhe o fenômeno/conteúdo, pois este está previamente implementado no computador, mas pode compreender $\mathrm{o}$ fenômeno/conteúdo implementado por meio da análise das simulações com base nas entradas informadas. 
Muitos softwares educativos já foram desenvolvidos visando a aprendizagem de conteúdo específicos do ensino infantil, fundamental e médio. Dada a ampla variedade de cursos superiores e o alto nível de conhecimento requerido para o desenvolvimento, ainda é bastante escasso o uso de softwares educativos nas universidades. No entanto, são apresentados a seguir alguns que tiveram significativo sucesso não apenas por tratar corretamente conteúdos de cursos de graduação, mas também pela utilização de simuladores.

Coelho et al. (2019) utilizaram como ferramenta didática um simulador de problemas envolvendo processos industriais da engenharia química, no Instituto Federal da Bahia (IFBA), chamado PRO/II. A justificativa consistiu no contato insuficiente dos estudantes com softwares computacionais durante a graduação e que hoje esta é uma habilidade imprescindível a qualquer engenheiro. Professores e alunos foram capacitados por meio de um guia tutorial. As simulações permitiram uma visão mais sistêmica dos resultados e o material gerado tem sido utilizado como uma ferramenta multiplicadora de conhecimento e como apoio didático em disciplinas específicas do curso de engenharia química.

Silva et al. (2019) desenvolveram um software educacional para o aprimoramento do ensino na disciplina de resistência dos materiais do curso de engenharia civil por meio do auxílio do software MATLAB. Nele o estudante pode simular diferentes tipos de situações as quais são demonstradas por meio da plotagem de imagens e gráficos que facilitam a compreensão do conteúdo abordado.

Siqueira e Fontes (2019) empregaram ferramentas de simulação fluidodinâmica e de análise estrutural por elementos finitos em aulas das turmas dos anos finais do curso de engenharia mecânica na Universidade São Judas Tadeu. Elas aumentaram a sensibilidade do aspecto físico dos problemas e desta forma ajudando no aprendizado de disciplinas fundamentais do curso e proporcionando a capacitação necessária para o desenvolvimento dos trabalhos de conclusão de curso.

$\mathrm{Na}$ Tabela 1 pode ser observado um comparativo entre os softwares educativos apresentados e a primeira versão do aplicativo Gravity Dams:

Tabela 1. Comparativo entre softwares educativos de simulação

\begin{tabular}{|c|c|c|c|c|c|}
\hline \multirow{2}{*}{ Autores } & Softwares & $\begin{array}{c}\text { Criação e } \\
\text { manutenção } \\
\text { de usuário, } \\
\text { uso de login }\end{array}$ & $\begin{array}{c}\text { Visualização } \\
\text { de conceitos } \\
\text { teóricos } \\
\text { diretamente } \\
\text { na aplicação }\end{array}$ & $\begin{array}{c}\text { Obtenção } \\
\text { de } \\
\text { feedback e } \\
\text { simulação }\end{array}$ & $\begin{array}{c}\text { Exercícios de } \\
\text { simulação da } \\
\text { realidade }\end{array}$ \\
\hline Coelho et al. & PRO/II & Não & Não & Sim & Sim \\
\hline Silva et al. & Matlab & Não & Não & Sim & Sim \\
\hline Siqueira e Fontes & ANSYS® & Não & Não & Sim & Sim \\
\hline \multicolumn{2}{|c|}{ Gravity Dams } & Sim & Sim & Sim & Sim \\
\hline
\end{tabular}

Barragens a gravidade são elementos importantes em diversas aplicações de engenharia. Esse aprendizado pode, e deve, ser auxiliado por novas tecnologias para ajuda ao aluno, e o software educativo proposto neste trabalho é de grande valia como 
agregador de fácil acesso para os conteúdos mais importantes, e como ferramenta de auxílio ao cálculo.

\section{Metodologia de Desenvolvimento do Aplicativo}

Há três metodologias com larga aplicação e citações na área que merecem destaque para elaboração de um modelo de simulação didático, a constar: Banks et al. apud Rangel (2015), Law apud Rangel (2015) e Rangel (2015). A partir da análise destas metodologias, suas semelhanças e divergências, a abordagem escolhida foi a de Rangel (2015), visto que apresenta a proposta que melhor representa o presente trabalho por focar na animação como recurso didático.

A metodologia direta para simulação proposta por Rangel (2015) é composta das seguintes etapas: (1) formulação e análise do conteúdo; (2) análise de viabilidade e definição dos objetivos; (3) modelo conceitual; (4) modelo computacional com animação; (5) testes com o modelo; (6) documentação e implementação.

Seguindo as etapas da metodologia de Rangel (2015), adotou-se para a metodologia de desenvolvimento do aplicativo Gravity Dams a seguinte estruturação:

1. Formulação e análise de conteúdo: corresponde ao levantamento da necessidade de abordar o conteúdo por meio de software. Neste trabalho esta etapa foi realizada por meio de pesquisa bibliográfica e análise do desempenho dos acadêmicos das engenharias no ENADE.

2. Análise de viabilidade e definição dos objetivos: corresponde ao estudo sobre a viabilidade da aplicação do software em sala de aula (infraestrutura), além da definição do objetivo a ser atingido com o software. Neste trabalho esta etapa foi realizada por meio da análise de pesquisas que tem como objetivo aprimorar o processo de ensino-aprendizagem em cursos de engenharia e entrevista com professores na área da engenharia civil.

3. Modelo conceitual: tem o objetivo de traduzir os conceitos do sistema que se pretende modelar, dando a ele uma estrutura que irá orientar e facilitar sua transição para a modelagem computacional. Esta modelagem foi realizada utilizando a linguagem gráfica UML (Unified Modeling Language) com os seguintes diagramas: caso de uso, classe e sequência.

4. Modelo computacional com animação: é a tradução do conteúdo teórico modelado na forma conceitual para um software. Para tanto, foi utilizado o seguinte ambiente de desenvolvimento: Android Studio; linguagem de programação Java 8.0; armazenamento de dados Google Cloud (Firebase).

5. Testes com o modelo: esta etapa tem como propósito validar a qualidade e a eficácia do modelo, a fim de verificar sua capacidade em representar os conceitos e a lógica do conteúdo teórico proposto. Também é verificado se as etapas representadas no modelo conceitual estão coerentes com as programadas no modelo computacional. Além disto, é testada a qualidade visual da animação construída a partir do modelo. Os testes foram realizados com a participação de um professor da área de engenharia civil e um profissional de desenvolvimento de software. As primeiras impressões do aplicativo Gravity Dams são apresentadas na seção 5 deste artigo. 
6. Documentação e implementação: corresponde a um relatório composto pelo modelo conceitual e o passo a passo da tradução para a forma computacional, com o objetivo de relatar detalhes do seu desenvolvimento, permitindo sua reprodução por outro programador ou uma futura modificação. A rigor, corresponde à totalidade do trabalho de conclusão de curso a qual este artigo está vinculado, visto que todas as etapas do desenvolvimento estão descritas de forma a permitir sua reprodução por terceiros. A documentação completa está disponível no Sistema de Bibliotecas da Universidade Federal do Paraná.

As características gerais do aplicativo Gravity Dams, considerando os requisitos indicados por Oliveira (2001), estão descritas na Tabela 2.

Tabela 2. Informações gerais do software educativo

\begin{tabular}{|l|l|}
\hline Nível de Ensino: & Superior em Engenharia Civil \\
\hline Ano/Período/Série: & Variável (5ํao 8o período) \\
\hline Disciplina: & Engenharia de Recursos Hídricos \\
\hline Conteúdo: & Estabilidade de Barragens à gravidade \\
\hline Corrente Pedagógica (Teoria Educacional): & Escola Nova \\
\hline Teoria de Aprendizagem: & Construtivista \\
\hline $\begin{array}{l}\text { Tipo de Objeto(s) de Aprendizagem } \\
\text { (classificação para Software Educativo): }\end{array}$ & Simulador \\
\hline Inclui Educação Inclusiva? & Não \\
\hline
\end{tabular}

\section{Apresentação do Aplicativo Gravity Dams}

O aplicativo Gravity Dams inicia apresentando uma história interativa na qual o objetivo é ajudar o castor Dandan (mascote) na construção de uma barragem, visando gerar hidroeletricidade. $\mathrm{O}$ andamento desta obra é mostrado na parte superior da tela de visualização dos módulos. A cada módulo completado a obra avança para próxima fase, conforme Figura 2.
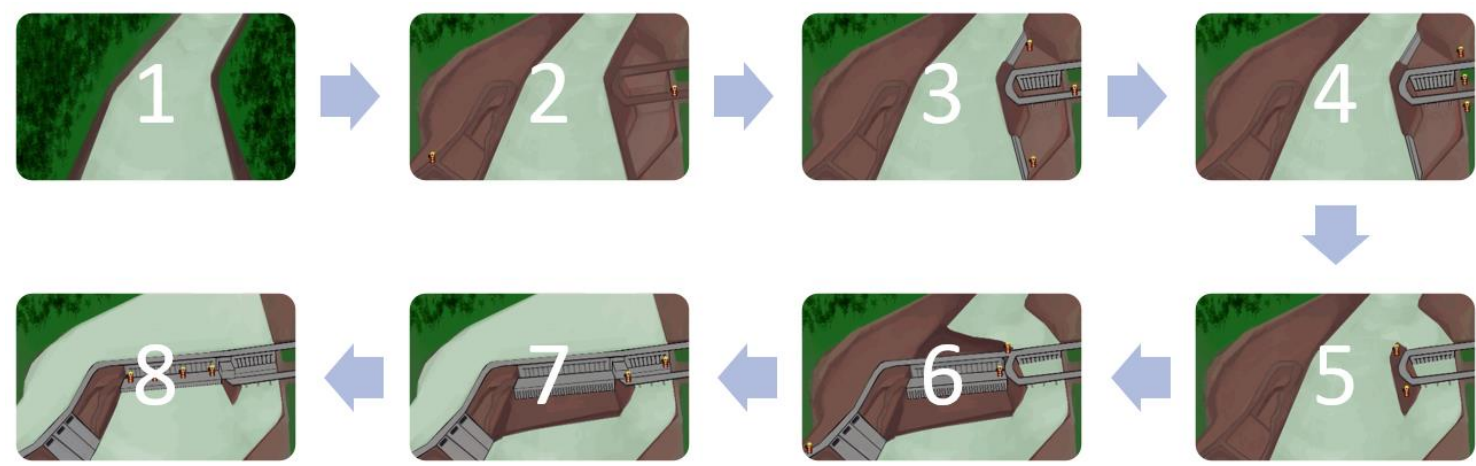

Figura 2. Níveis da obra da usina hidrelétrica.

Os principais passos a serem seguidos na interação com o aplicativo Gravity Dams nas simulações de estabilidade (flutuação, tombamento, deslizamento e tensões na base) são os seguintes:

1. Pré-configuração (opcional): alteração dos coeficientes mínimos padrões para cada caso de carregamento e para cada verificação (flutuação, tombamento e 
deslizamento), os quais não podem ser inferiores a 1 (um). Além disso, informase a porcentagem mínima de área da base que deverá estar comprimida para cada caso de carregamento;

2. Seleção da seção de estudo da barragem e inserção das respectivas dimensões geométricas, conforme o Sistema Internacional de Unidades;

3. Definição dos pesos específicos e parâmetros geomecânicos;

4. Definição das respectivas alturas dos níveis de água (montante e jusante) e do caso de carregamento correspondente;

5. Por fim, o aluno deverá efetuar os cálculos das variáveis solicitadas e, posteriormente, verificar simulação do resultado.

Para apresentação do software educativo, foi testado seu uso em um dispositivo com sistema operacional Android 10 (API 29), com um novo usuário, considerando os fluxos principais dos casos de usos. Na Figura 3 é mostrado um fluxograma do caminho principal que pode ser percorrido no software.

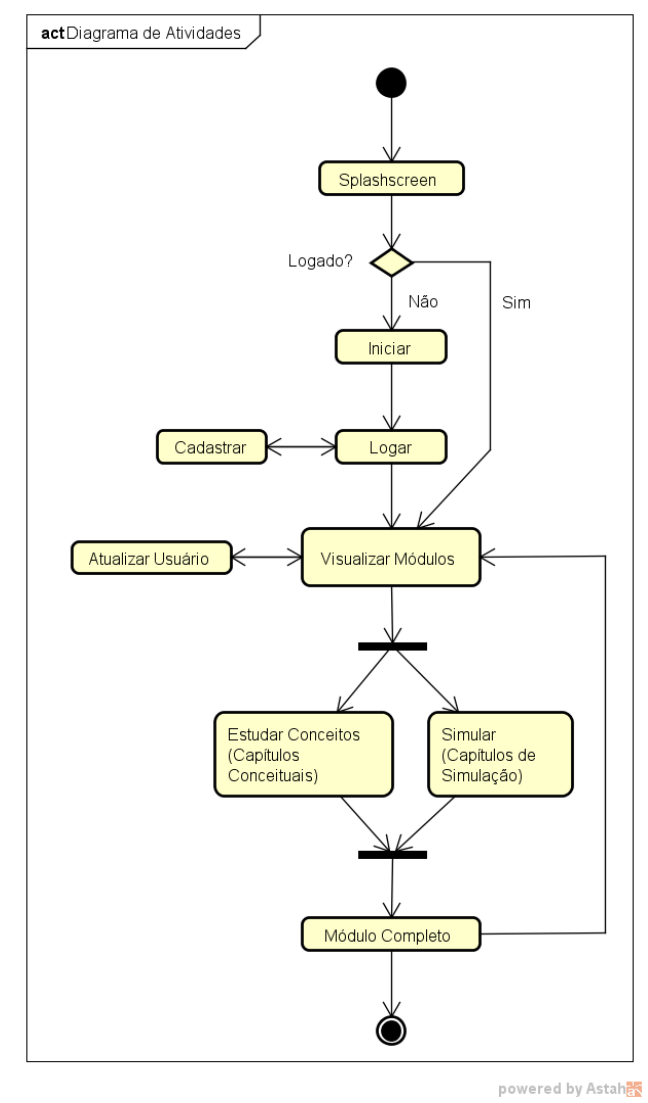

Figura 3. Fluxograma do caminho principal percorrido no software

Este teste contemplou a seguinte sequência:

1. Splashscreen, iniciar e logar (Figura 4);

2. Guia de apresentação inicial: módulos e configurações (Figura 5);

3. Capítulo conceitual (Figura 6);

4. Capítulo de simulação (Figura 7). 


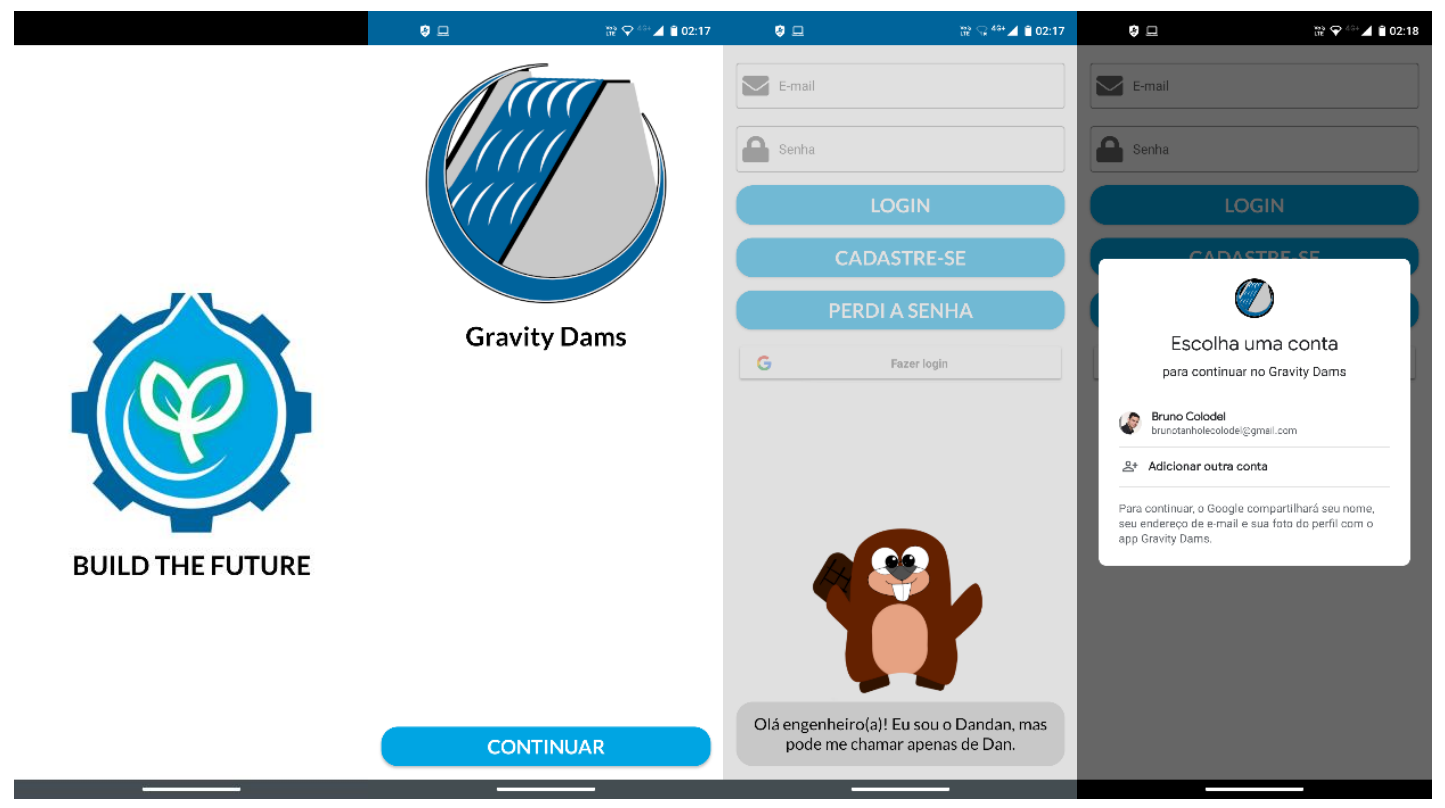

Figura 4. Sequência de prints das telas iniciais.

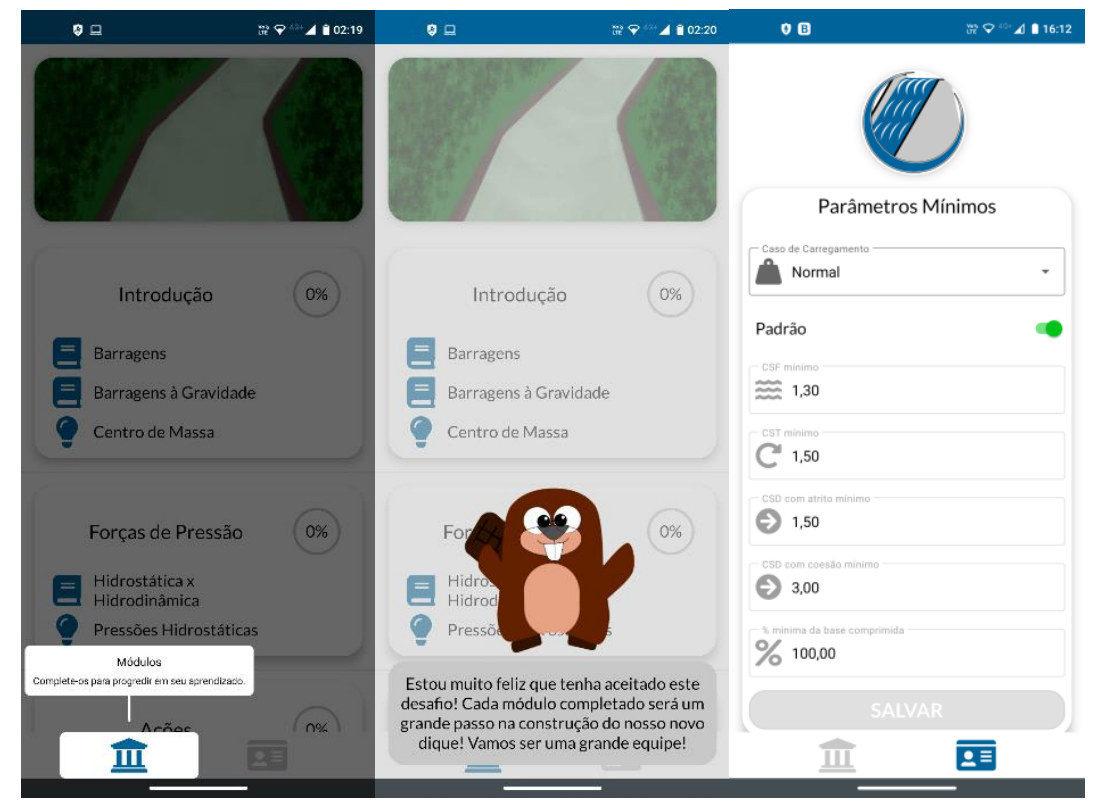

Figura 5. Sequência de prints da introdução após login. 


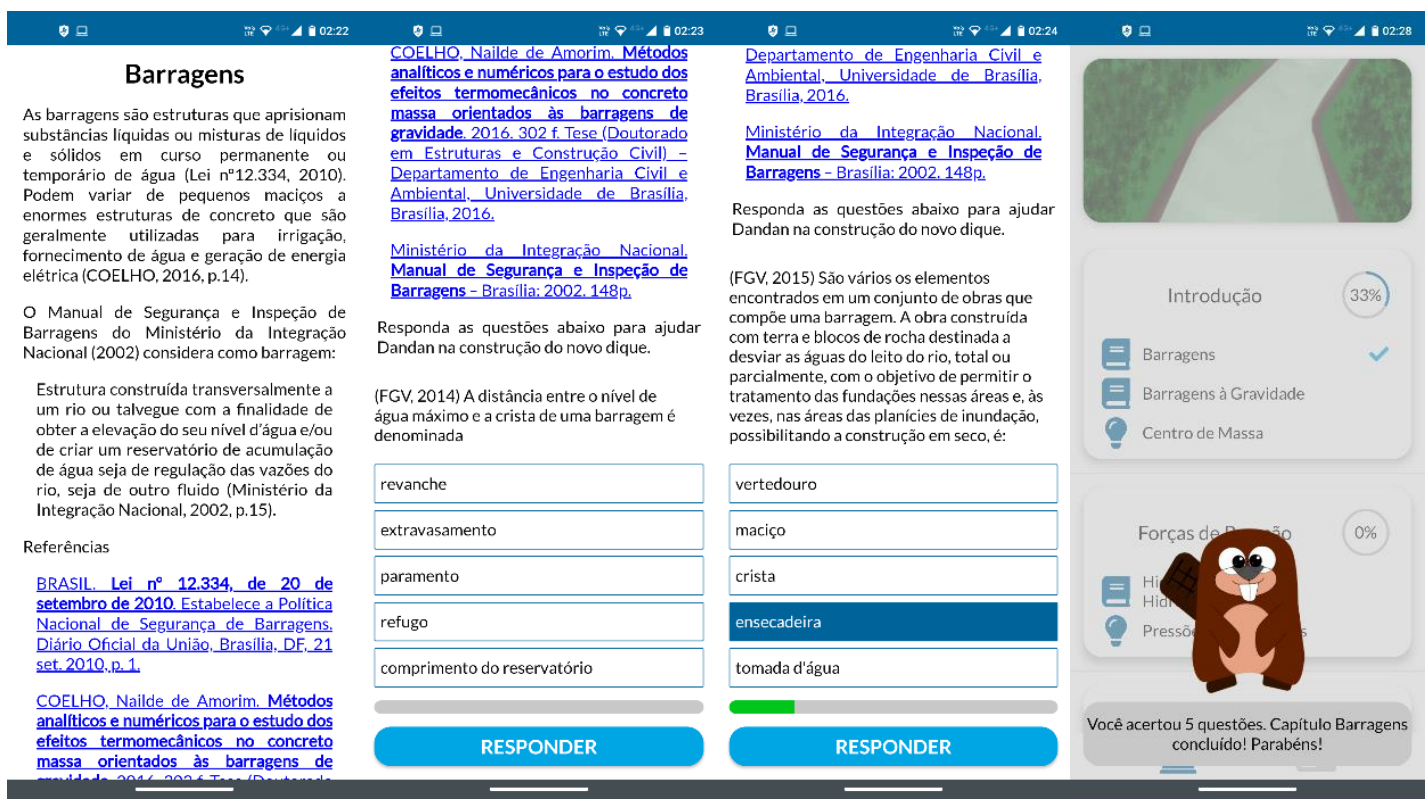

Figura 6. Sequência de prints em capítulo conceitual.

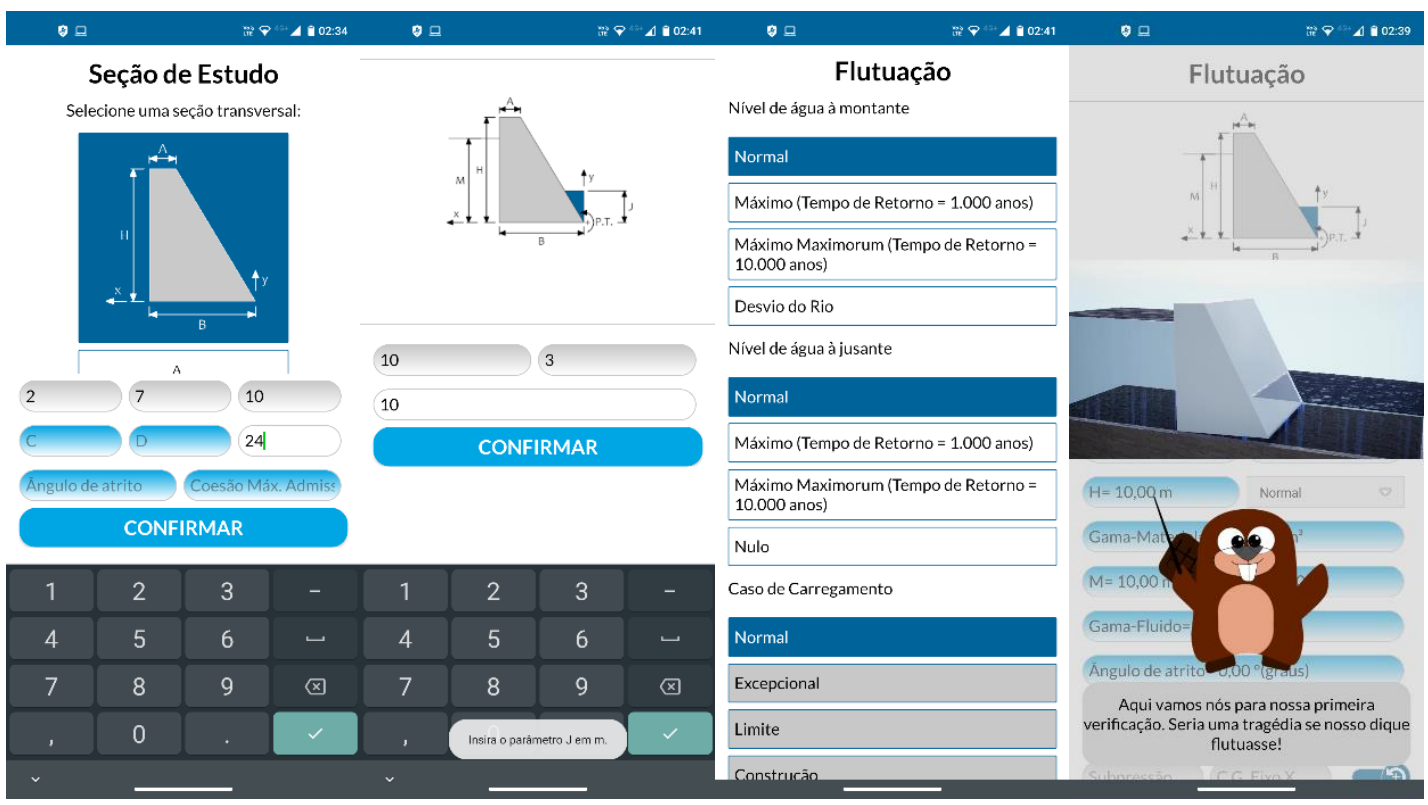

Figura 7. Sequência de prints da simulação de flutuação.

\section{Primeiras Impressões do Aplicativo Gravity Dams}

A avaliação de um software educativo pode ocorrer de duas maneiras principais: pela visão do professor ou pela visão do analista (podendo também ser avaliado pela visão do aluno).

A avaliação pela visão do professor tem o objetivo de definir o melhor objeto de aprendizagem para um conteúdo específico de forma autoexplicativa, sem a necessidade de conhecimentos aprofundados em informática. Para tanto, Raetegui, Boff e Finco (2010) definiram diretrizes nas quais os softwares são avaliados pedagogicamente e tecnicamente. 
Já na visão do analista a avaliação é feita de forma técnica realçando conceitos de engenharia de software e ergonomia. Portanto, é uma avaliação que permite que projetistas definam requisitos com facilidade para alcançar a qualidade esperada. Oliveira (2001) propôs um método avaliativo que verifica a apresentação e funcionalidades, a confiabilidade, eficiência, manutenibilidade, portabilidade, usabilidade e o nível de correlação com o conteúdo a ser estudado. Por meio da soma das pontuações obtidas (máximo de 187 pontos) o software é classificado como insatisfatório, regular, bom ou excelente.

Após a implementação do aplicativo Gravity Dams foram feitas uma avaliação pela visão do professor, conforme Raetegui, Boff e Finco (2010) e uma avaliação pela visão do analista, conforme Oliveira (2001). As avaliações foram solicitadas para um professor especialista no assunto abordado pelo software e para um programador de computadores.

Na visão do analista o aplicativo Gravity Dams foi classificado como excelente, atingindo a pontuação de 155 pontos de 187 possíveis. O quesito com menor pontuação foi "Portabilidade" devido ao software educativo ser executado somente em sistema operacional Android. Já na visão do professor especialista o aplicativo Gravity Dams obteve a pontuação de 29 de 34 possíveis. Os dois requesitos considerados importantes para o professor, mas que não foram implementados no aplicativo são: (1) possibilidade de registro e consulta as ações desenvolvidas o que permitiria aos alunos e alunas reverem e retomarem o seu processo de construção do conhecimento; (2) não dispõe de ferramentas de comunicação que permitem a interação entre os estudantes e o professor.

\section{Considerações Finais}

O objetivo deste artigo foi apresentar o desenvolvimento da primeira versão do aplicativo Gravity Dams para o ensino de estabilidade de barragens à gravidade. O desenvolvimento dessa ferramenta buscou disponibilizar um software educativo para auxiliar no processo de ensino-aprendizagem de conceitos acerca de estabilidade de seções com largura unitária e comuns de barragens à gravidade, demonstrando o passo a passo das análises e simulações decorrentes de cada resultado encontrado. Trata-se de uma ferramenta complementar ao trabalho do professor em sala de aula.

Todo o material elaborado neste trabalho é o passo inicial na criação de uma série de aplicações intitulada "Build the Future", com o intuito de auxiliar o ensino de engenharia em novos tópicos. O aprendizado adquirido com a elaboração do Gravity Dams foi, portanto, fundamental para identificar os conhecimentos necessários para o desenvolvimento dos próximos softwares da série.

\section{Referências}

Abreu, L. C. et al. (2010) “A epistemologia genética de Piaget e o construtivismo”. Em Revista Brasileira Crescimento e Desenvolvimento Humano, p. 361-366.
Brasil
(2010),
Lei $\quad \mathrm{n}^{\circ}$
12.334 .
Disponível
em: $<$ http://www.planalto.gov.br/ccivil_03/_ato2007-2010/2010/lei/112334.htm>. Acesso em Julho, 2021. 
Coelho, N. A. (2016) "Métodos analíticos e numéricos para o estudo dos efeitos termomecânicos no concreto massa orientados às barragens de gravidade". Tese de Doutorado, Universidade de Brasília, Brasília, Brasil.

Coelho, M. S. et al. (2019) "Uso do simulador PRO/II como ferramenta didática auxiliar no curso de engenharia química do IFBA". Em Engenharia no século XXI, vol. 2, p. 155-163, Editora Poisson. Disponível em: $<$ https://www.poisson.com.br/livros/engenharia/volume2/ESEC2.pdf $>$. Acesso em Agosto, 2020.

Eletrobrás (2003) "Critérios de Projeto Civil de Usinas Hidrelétricas". Eletrobrás/CBDB, Brasília, Brasil.

Ministério da Educação (2019) "Relatório do ENADE 2019”, Brasília, Brasil.

Oliveira, N. (2001) "Uma proposta para avaliação de software educacional". Dissertação de Mestrado, Universidade Federal de Santa Catarina, Florianópolis, Brasil.

Pereira, M. A. (2005) "Ensino-Aprendizagem em um Contexto Dinâmico - O Caso de Planejamento de Transportes". Tese de Doutorado, Universidade de São Paulo, São Carlos, Brasil.

Raetegui, E. Boff, E. Finco, M. D. (2010) "Proposta de diretrizes para avaliação de objetos de aprendizagem considerando aspectos pedagógicos e técnicos". Centro Interdisciplinar de Novas Tecnologias na Educação, Universidade Federal do Rio Grande do Sul, Porto Alegre, Brasil.

Rangel, J. J. A. (2015) "Uma abordagem para construção de modelos de simulação a eventos discretos para aplicação como um recurso didático". Em Revista Produto \& Produção, vol. 16 n.2, p. 56-80, jun. 2015.

Silva, P. B. et al. (2019) "Desenvolvimento de software educacional para aprimoramento do ensino da resistência dos materiais. Engenharia no século XXI", vol. 2, p. 181-188, Editora Poisson. Disponível em: $<$ https://www.poisson.com.br/livros/engenharia/volume2/ESEC2.pdf $>$. Acesso em Agosto, 2020.

Siqueira, C. R. Fontes, C. E. (2019) "Utilização de simulação numérica para auxílio do ensino de engenharia e apoio a projetos de TCC". Em Engenharia no século XXI, vol. 2, p. 197-205, Editora Poisson. Disponível em: $<$ https://www.poisson.com.br/livros/engenharia/volume2/ESEC2.pdf $>$. Acesso em Agosto, 2020.

Trevisan, D. Silva, H. R. A. S. (2019) “Aplicação do método de ensino Peer Instruction no curso de Engenharia Civil em disciplinas de cálculo estrutural”. Em Engenharia no século XXI, vol. 2, p. 73-80, Editora Poisson. Disponível em: $<$ https://www.poisson.com.br/livros/engenharia/volume2/ESEC2.pdf $>$. Acesso em Agosto, 2020.

Valente, J. A. (1999), O Computador na Sociedade do Conhecimento. Campinas, São Paulo, Brasil. UNICAMP/NIED. 